\title{
Boron Hyperfine Structure in Trifluorophosphine-Borane
}

W. Kasten and H. Dreizler

Abteilung Chemische Physik im Institut für Physikalische Chemie der Universität Kiel

R. L. Kuczkowski

Department of Chemistry, University of Michigan, Ann Arbor, MI 48109

Z. Naturforsch. 40a, 920-922 (1985); received July 17, 1985

We reinvestigated the microwave spectrum of trifluorophosphine-borane by microwave Fourier transform spectroscopy and determined the quadrupole coupling and centrifugal distortion constants for both isotopes ${ }^{10} \mathrm{~B}$ and ${ }^{11} \mathrm{~B}$. The $\mathrm{B}-\mathrm{P}$ bond order is discussed.

The microwave spectrum of trifluorophosphineborane, $\mathrm{PF}_{3} \mathrm{BH}_{3}$, was first investigated by Kuczkowski and Lide [1]. We reinvestigated the spectrum with the higher resolution of microwave Fourier transform (MWFT) spectroscopy to resolve the boron-hfs. It is the first time that the boron-hfs of an addition compound of boron and an element of the fifth group of the periodic table has been resolved. It will be interpreted in terms of the B-P bonding.

The sample was prepared according to [1] and measured at temperatures around $-50^{\circ} \mathrm{C}$ and pressures around $0.5 \mathrm{~m}$ Torr. The MWFT-spectrometers are described in $[2,3]$. The $J=1-0$ and $J=2-1$ transitions of the ${ }^{10} \mathrm{~B}$ compound with nuclear spin $I=3$ and of the ${ }^{11} \mathrm{~B}$ compound with $I=3 / 2$ were investigated in natural abundance [4]. The measurements are given in Tables 1 and 2. A recording is given in Figure 1. The frequencies were determined by a line shape analysis [5].

First the quadrupole coupling constants $e q Q$ were determined from the $J=1-0$ transitions. For the $J=2-1$ transitions the assignment was complicated by the hfs and $K$ fine structure. The two strongest components of each multiplet were assigned to the $F=5-4$ for ${ }^{10} \mathrm{~B}$ and $F=7 / 2-5 / 2$ for ${ }^{11} \mathrm{~B}$ with the $K=0$ and $|K|=1$ assignment unknown. Considering the squared dipole matrix elements for $K=0$ and $K=1$ transitions one gets [6]:

$$
\mu_{21}(K=0)^{2}: \mid \mu_{21}(K=1)^{2}=4: 3 .
$$

The intensity ratio is modified by the $K$-degeneracy and statistical spin weight to $4: 5$ assuming $\mathrm{C}_{3 v^{-}}$

Reprint requests to Prof. Dr. H. Dreizler, Abteilung Chemische Physik im Institut für Physikalische Chemie der Universität Kiel, Olshausenstr. 40, D-2300 Kiel. symmetry of the molecule. Thereby the $K$ assignment for ${ }^{10} \mathrm{~B}$ was possible as given in Table 1. For ${ }^{11} \mathrm{~B}$ the intensity argument was useless as $K=0$, $F=7 / 2-5 / 2$ and $F=5 / 2-3 / 2$ overlap which gives nearly the same intensity as $K= \pm 1, F=7 / 2-5 / 2$. We favoured an assignment which resulted in the

Table 1. Measured frequencies $v_{\mathrm{obs}}$ of trifluorophosphineborane- $\left({ }^{10} \mathrm{~B}\right)$ refined by line shape analysis. $v_{\text {calc }}$ calculated with constants of Table 3 . $v_{\text {unsplit }}$ hypothetical frequency without hfs-splitting (see text). Frequencies in $\mathrm{MHz}$.

\begin{tabular}{llllll}
\hline$J^{\prime}-J$ & $K$ & $F^{\prime}-F$ & \multicolumn{1}{l}{$v_{\text {obs }}$} & \multicolumn{1}{l}{$v_{\text {calc }}$} & $v_{\text {unsplit }}$ \\
\hline $1-0$ & 0 & $3-3$ & 7847.317 & 7847.317 & \\
& & $4-3$ & 7846.668 & 7846.668 & 7846.830 \\
& & $2-3$ & 7846.441 & 7846.441 & \\
$2-1$ & 0 & $5-4$ & 15693.564 & 15693.564 & \\
& & $4-3$ & - & 15693.494 & 15693.633 \\
& & $3-3$ & - & 15693.377 & \\
& \pm 1 & $5-4$ & 15693.412 & 15693.412 & \\
& & $4-3$ & $15693.989 *$ & 15694.026 & 15693.609 \\
& & $3-3$ & $15693.989 *$ & 15693.980 & \\
\hline
\end{tabular}

* not used.

Table 2. Measured frequencies of trifluorophosphineborane- $\left({ }^{11} \mathrm{~B}\right)$. See Table 1.

\begin{tabular}{llllll}
\hline$J^{\prime}-J$ & $K$ & \multicolumn{1}{l}{$F^{\prime}-F$} & \multicolumn{1}{l}{$v_{\text {obs }}$} & \multicolumn{1}{l}{$v_{\text {calc }}$} & \multicolumn{1}{l}{$v_{\text {unsplit }}$} \\
\hline $1-0$ & 0 & $3 / 2-3 / 2$ & 7614.400 & 7614.400 & \\
& & $5 / 2-3 / 2$ & 7614.030 & 7614.030 & 7614.104 \\
& & $1 / 2-3 / 2$ & 7613.735 & 7613.735 & \\
$2-1$ & 0 & $7 / 2-5 / 2$ & 15228.149 & 15228.149 & \\
& & $5 / 2-3 / 2$ & 15228.149 & 15228.149 & 15228.180 \\
& & $3 / 2-3 / 2$ & - & 15227.884 & \\
& \pm 1 & $7 / 2-5 / 2$ & 15228.068 & 15228.068 & \\
& & $5 / 2-3 / 2$ & - & 15228.438 & 15228.158 \\
& & $3 / 2-3 / 2$ & - & 15228.306 & \\
\hline
\end{tabular}


same sign of the centrifugal distortion parameter $D_{J K}$ for both isotopes. By addition of the hfs-corrections to the components of the multiplets $v_{\text {unsplit }}$ was calculated as a mean value. These frequencies were used to determine the rotational constant $B$ and the centrifugal distortion constants $D_{J}$ and $D_{J K}$ [7] given in Table 3 together with eq $Q$.

Next we interpret the quadrupole coupling constants in terms of the bond order following Townes and Dailey [8] and Gordy [9]. For details see [4]. The following equation is used

$$
e Q q=-(\mathrm{Up})_{z} \cdot \frac{e Q q_{210}}{1+\left(n-3 i_{\sigma}\right) \cdot \varepsilon},
$$

where $e Q q$ is the measured quadrupole coupling constant, $e Q q_{210}\left({ }^{10} \mathrm{~B}\right)=-11.83 \mathrm{MHz}, e Q q_{210}\left({ }^{11} \mathrm{~B}\right)$ $=-5.39 \mathrm{MHz}[10]$ are the quadrupole coupling con-

Table 3. Rotational, centrifugal distortion and quadrupole coupling constants of trifluorophosphine-borane- $\left({ }^{10} \mathrm{~B}\right)$ and $-\left({ }^{11} \mathrm{~B}\right)$. Standard deviation in brackets in units of the last digit. For $B, D_{J}$, and $D_{J K}$ no error calculation is possible as only three frequencies are available.

\begin{tabular}{|c|c|c|c|}
\hline & $\mathrm{PF}_{3}{ }^{10} \mathrm{BH}_{3}$ & & {$[1]$} \\
\hline \multirow[t]{2}{*}{$\begin{array}{l}B= \\
D_{J}= \\
D_{J K}= \\
e Q q=\end{array}$} & $\begin{array}{ll}= & 3923.4173 \\
= & 1.13 \\
= & 6.03 \\
= & 3.2445(7)\end{array}$ & $\begin{array}{l}\mathrm{MHz} \\
\mathrm{kHz} \\
\mathrm{kHz} \\
\mathrm{MHz}\end{array}$ & $3923.389(50) \mathrm{MHz}$ \\
\hline & $\mathrm{PF}_{3}{ }^{11} \mathrm{BH}_{3}$ & & [1] \\
\hline $\begin{array}{l}B= \\
D_{J}= \\
D_{J K}= \\
e Q q=\end{array}$ & $\begin{array}{ll}= & 3807.0543 \\
= & 1.15 \\
= & 5.68 \\
= & 1.478(3)\end{array}$ & $\begin{array}{l}\mathrm{MHz} \\
\mathrm{kHz} \\
\mathrm{kHz} \\
\mathrm{MHz}\end{array}$ & $\begin{array}{c}3807.067(50) \mathrm{MHz} \\
1.1(8) \\
\mathrm{kHz}\end{array}$ \\
\hline
\end{tabular}

stants induced by a $2 \mathrm{p}_{z}$-electron, $\varepsilon=0.5$ [11] is the shielding constant for boron, $i_{\sigma}=\frac{1}{2}|E N(\mathrm{H})-E N(\mathrm{~B})|$ is the ionic character of the $\mathrm{B}-\mathrm{H}$ bond calculated from the electronegativities $E N(\mathrm{H})=2.15$ and $E N(\mathrm{~B})=2.0$ [12], $(\mathrm{Up})_{z}$ is the number of "unbalanced" p-electrons in $z$ direction, i.e. the $\mathrm{B}-\mathrm{P}$ bond direction and $n$ is the number of electrons donated by the phosphorus to boron; $n$ is the aim of this interpretation. $(\mathrm{Up})_{z}$ may be calculated from

$$
\begin{aligned}
(\mathrm{Up})_{z}=- & \frac{3}{2}\left(1-i_{\sigma}\right) \cdot a_{\mathrm{p}}^{2}\left(\psi_{\mathrm{H}}\right) \\
& \cdot\left(3 \cos ^{2}(\Varangle(\mathrm{PBH}))-1\right)-n \cdot a_{\mathrm{p}}^{2}\left(\psi_{\mathrm{p}}\right),
\end{aligned}
$$

where $a_{\mathrm{p}}^{2}\left(\psi_{\mathrm{H}}\right)$ is the p-electron fraction of the boron orbital directed to the hydrogen occupied by $\left(1-i_{\sigma}\right)$ electrons, $a_{\mathrm{p}}^{2}\left(\psi_{\mathrm{p}}\right)$ is the fraction of the boron orbital $\psi_{\mathrm{p}}$ directed to the phosphorus occupied by $n$ electrons and $\Varangle(\mathrm{PBH})=103(1)^{\circ}$ is the bond angle known from the structure [1].

Approximating $\psi_{\mathrm{H}}=s_{\mathrm{H}}+\lambda p_{\mathrm{H}}$ by a hybridisation mixing of $s$ and $p$ functions [13] results in $a_{\mathrm{p}}^{2}\left(\psi_{\mathrm{H}}\right)$ as:

$$
a_{\mathrm{p}}^{2}\left(\psi_{\mathrm{H}}\right)=\frac{\lambda^{2}}{1+\lambda^{2}} ; \quad \lambda^{2}=-\{\cos (\Varangle(\mathrm{HBH}))\}^{-1},
$$

where $\Varangle(\mathrm{HBH})=115(1)^{\circ}$ from the structure [1].

In a similar way

$$
a_{\mathrm{p}}^{2}\left(\psi_{\mathrm{p}}\right)=\frac{\mu^{2}}{1+\mu^{2}} ; \mu^{2}=\{\lambda \cdot \cos (\Varangle(\mathrm{PBH}))\}^{-2} .
$$

The information is sufficient to determine for both isotopes the bond order $n=0.57$ (see Fig. 2), which is the first determined by microwave spectroscopy for a III-V addition compound and similar to the value 0.53 determined for $\mathrm{BH}_{3} \mathrm{CO}$ [9].
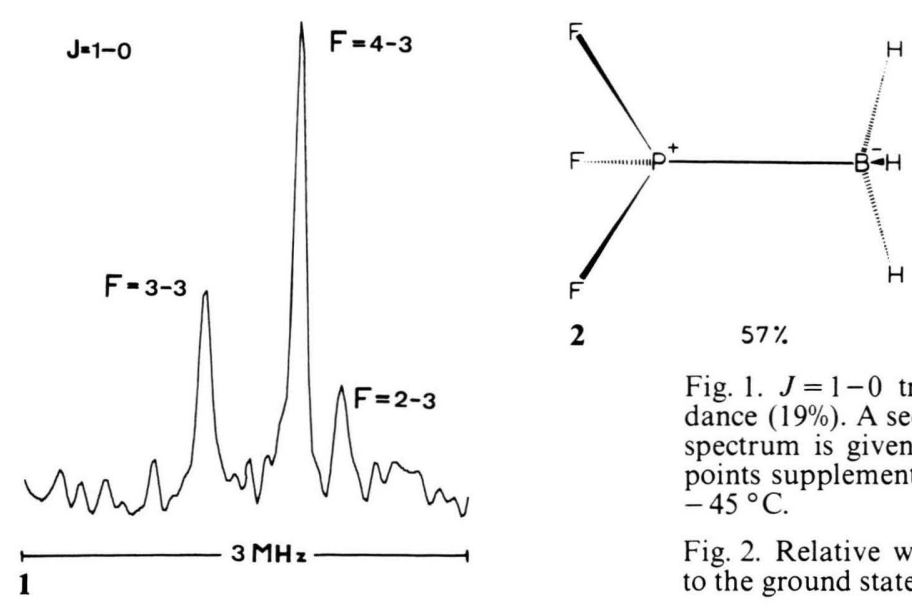

2
$57 \%$

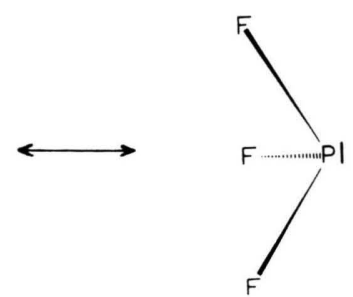

$43 \%$

Fig. 1. $J=1-0$ transition of $\mathrm{PF}_{3}{ }^{10} \mathrm{BH}_{3}$ measured in natural abundance $(19 \%)$. A section of $3 \mathrm{MHz}$ out of a $50 \mathrm{MHz}$ range of the power spectrum is given. Sample interval $10 \mathrm{~ns}, 12800 \mathrm{k}$ cycles, 1024 data points supplemented by 3072 zeros, pressure $0.8 \mathrm{mTorr}$, temperature $-45^{\circ} \mathrm{C}$.

Fig. 2. Relative weight of the principal structures, which contribute to the ground state of trifluorophosphine-borane. 
The authors thank Dr. D. Boseth for assistance in the preparation of the compound. W. K. acknowledges a scholarship of the Fonds der Chemie, R. L. K. of a travel grant to Kiel from the Rackham

[1] R. L. Kuczkowski and D. R. Lide, J. Chem. Phys. 46, 357 (1967)

[2] G. Bestmann, H. Dreizler, E. Fliege, and W. Stahl, J. Mol. Struct. 97, 215 (1983)

[3] G. Bestmann and H. Dreizler, Z. Naturforsch. 37a, 58 (1982).

[4] W. Kasten, Thesis, Kiel 1985.

[5] E. Fliege and H. Dreizler, Z. Naturforsch. 39a, 630 (1984).

[6] C. H. Townes and A. L. Schawlow, Microwave Spectroscopy, McGraw Hill, London 1955, Equation $3-40$.
Graduate School, University of Michigan. We thank the Deutsche Forschungsgemeinschaft and Fonds der Chemie for funds. The calculations were made at the computer center of the University of Kiel.

[7] 1.c. [6], Equation 3-55.

[8] 1.c. [6], Chapter 9.

[9] W. Gordy and R. L. Cook, Microwave Molecular Spectra, J. Wiley, New York, Chapter XIV.

[10] 1.c. [9], Table 14.2.

[11] 1.c. [9], Table 14.3.

[12] 1.c. [9], Equation (14.93) and Appendix G.

[13] C. A. Coulson, Die Chemische Bindung, Hirzel, Stuttgart 1969, p. 162, Equations (1) to (3). 